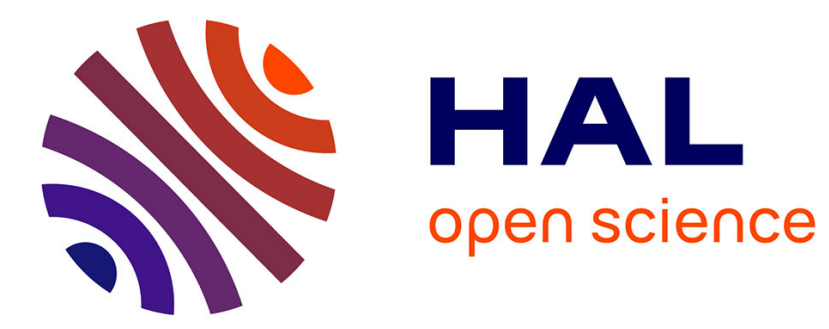

\title{
Je, tu, nous et les autres: le " versant subjectif " des essais d'Édouard Glissant
}

\author{
Florian Alix
}

\section{To cite this version:}

Florian Alix. Je, tu, nous et les autres: le "versant subjectif" des essais d'Édouard Glissant. Presence africaine (Paris, France: 1967), 2011, 184 (2), pp.33-52. 10.3917/presa.184.0033 . hal03279739

\section{HAL Id: hal-03279739 \\ https://hal.sorbonne-universite.fr/hal-03279739}

Submitted on 6 Jul 2021

HAL is a multi-disciplinary open access archive for the deposit and dissemination of scientific research documents, whether they are published or not. The documents may come from teaching and research institutions in France or abroad, or from public or private research centers.
L'archive ouverte pluridisciplinaire HAL, est destinée au dépôt et à la diffusion de documents scientifiques de niveau recherche, publiés ou non, émanant des établissements d'enseignement et de recherche français ou étrangers, des laboratoires publics ou privés. 


\section{Je, tu, nous et les autres : le « versant subjectif » des essais d'Edouard Glissant}

« On ne s'en sort pas, on ne s'exprime jamais, on reste toujours soi-même par les autres, on devient soi par autrui. » (Daniel Poliquin, L'Obomsawin)

L'écrivain antillais du milieu du XXème siècle, porté par la dynamique anticoloniale, faisait de sa voix l'écho d'une voix collective. Ainsi, Césaire, lorsqu'il remanie son Discours sur le colonialisme pour le publier chez Présence Africaine ${ }^{1}$, mêle le « je » caractéristique de la critique et du pamphlet à un «nous » de revendication collective. La voix de l'auteur se confond avec la voix politique des colonisés. Césaire retrouve cette posture énonciative dans sa Lettre à Maurice Thorez où il affirme « qu'aucune doctrine ne vaut que repensée par nous, que repensée pour nous, que convertie à nous $»^{2}$. L'auteur se fait alors le porte-parole de son peuple : il écrit au nom de la communauté qu'il représente.

La posture énonciative change pour les écrivains de la génération suivante, mais la tension avec le collectif semble bien perdurer. En effet, dans son étude sur la littérature antillaise des années 1980, Dominique Chancé constate que les écrivains refusent la posture de l'auteur, solitaire, mais leur rapport à la collectivité a changé cependant. Ils préfèrent selon elle la position de « marqueur de paroles » à celle d'auteur : " ils apparaissent dans leurs récits sous les traits de personnages dérisoires et maladroits, bricoleurs et nomades qui se mêlent à la foule babillarde, sans faire entendre clairement leur propre discours $»^{3}$. L'écrivain continue à évoquer une collectivité, mais il ne la considère plus dans sa globalité et s'attache à rendre compte de sa diversité, sur un mode fragmentaire. Il ne s'en fait plus le porte-parole, mais se présente à la fois comme son analyste et un de ses membres.

Qu'en est-il d'Edouard Glissant? Dominique Chancé voit en lui un «marqueur de paroles ", rappelant que Raphaël Confiant et Patrick Chamoiseau se revendiquent de lui dans leur pratique d'écriture ${ }^{4}$. En même temps, l'écrivain choisit aussi parfois une énonciation à la première personne du pluriel : il formule alors une parole collective, use d'un " nous » global plutôt qu'il ne consigne des échos venus de divers endroits du tissu social. Et que devient la parole individuelle de l'auteur dans cette alternative?

\footnotetext{
${ }^{1}$ Sur les aventures éditoriales du texte, voir R. Fonkoua, Aimé Césaire, Paris, Perrin, 2010, pp. 173-179.

${ }^{2}$ A. Césaire, Lettre à Maurice Thorez, in Euvre historique et politique, Fort-de-France, Désormeaux, 1976, p. 469.

${ }^{3} \mathrm{D}$. Chancé, L'Auteur en souffrance - Essai sur la position et la représentation de l'auteur dans le roman antillais contemporain, 1981-1992, Paris, PUF, 2000, p. 2.

${ }^{4}$ Ibid., p. 4.
} 
L'essai, un genre qu'affectionne particulièrement Glissant, permet de repenser la place de l'écrivain dans son œuvre. En effet, le genre présente selon Jean Starobinski «deux versants ", «l'un objectif, l'autre subjectif $»^{5}$. L'essai est un discours de savoir : il vise à établir une vérité qui a une valeur absolue, qui dépasse les communautés et les individus. Mais il est aussi un discours subjectif : il se caractérise par « [un] aspect réflexif, [un] versant subjectif $[\ldots]$ où la conscience de soi s'éveille comme une nouvelle instance de l'individu, instance qui juge l'activité du jugement, qui observe la capacité de l'observateur »6 ${ }^{6}$ L'essai oscillerait entre le discours gnomique et l'intimité de la première personne du singulier plutôt qu'il ne serait une écriture du «nous ». A l'extrême, il s'opposerait même à tout discours social : Scott Russell Sanders voit ainsi l'essai comme « un havre pour la voix privée, idiosyncratique dans une époque de babil anonyme $»^{7}$.

L'essai permet donc de relier la question du rapport de l'écrivain à une communauté à celle de l'écriture d'une subjectivité. Glissant n'est sans doute pas le porte-parole des Antillais ni le scribe d'un discours social éclaté ; il est l'auteur d'une œuvre originale où le sujet, le « je » qui écrit, s'interroge sur la construction et les évolutions des communautés antillaises dans le monde. L'essai organise un jeu d'échos et de tensions entre le «je », le «nous» et bien sûr le « tu » du lecteur.

L'essayiste adopte donc une certaine posture d'énonciation, à l'instar du romancier, même si celle-ci n'est pas fictive. L'écrivain de fiction fait parler un narrateur, suscitant une scène d'énonciation qui assigne une place codifiée au lecteur. Mais l'essayiste s'adresse lui aussi au lecteur d'une certaine manière en se positionnant par rapport à lui. On peut donc s'interroger sur la scénographie ${ }^{8}$ déployée par Glissant dans ses essais, sur la façon dont le texte organise les rapports entre le sujet de l'énonciation, le lecteur et le reste du monde. En effet, l'auteur du Discours antillais et de Poétique de la Relation met en scène, situe sa parole par rapport à son interlocuteur et par rapport à différentes instances collectives : l'essai construit le cadre énonciatif qui doit guider sa lecture.

Ce travail de scénographie amène Glissant à reconfigurer le sujet postcolonial, à transformer les relations entre la parole singulière et les discours collectifs. Ce faisant, il construit un lecteur idéal, mais il révèle aussi le rôle du lecteur dans la construction de la

\footnotetext{
5 J. Starobinski, « Peut-on définir l'essai ? », in F. Dumont (ed.), Approches de l'essai, Québec, Nota Bene, 2003, p. 171.

${ }^{6}$ Ibid., p. 174.

${ }^{7}$ S.R. Sanders, « The Singular First Person », in A.J. Butrym, Essays on the Essay - Redefining the genre, Athens (Ga) / Londres, University of Georgia Press, 1989, p. 33 (Nous traduisons).

${ }^{8}$ Sur ce concept, voir D. Maingueneau, Le Discours littéraire : paratopie et scène d'énonciation, Paris, Armand Colin - U, 2004, pp. 192-193.
} 
subjectivité de l'auteur. En reconfigurant la scénographie énonciative de l'essai, il recompose la fonction de l'auteur dans son texte.

\section{Le « je » et le « nous » : reconfiguration du sujet postcolonial}

\section{De la révélation à la médiation}

Entre le Discours sur le colonialisme (A. Césaire, 1955) et l'Eloge de la créolité (J. Barnabé, P. Chamoiseau, R. Confiant, 1989), la posture de l'écrivain a changé. Le sujet césairien s'engageait en même temps dans les lettres et dans le champ politique. Il se faisait volontiers le représentant de son peuple - ainsi Luciano C. Picanço voit dans le Cahier d'un retour au pays natal « un document contractuel à travers lequel le poète se doit d'assumer un rôle envers la population de son pays $»^{9}$. Il était le porte-voix de revendications collectives : le « nous » de Césaire renvoie à une communauté en quête de ses droits contre la puissance coloniale qui les lui dénie.

Le «nous » de Jean Barnabé, Patrick Chamoiseau et Raphaël Confiant n'est pas le même. Les auteurs d'Eloge de la créolité déplorent en effet la tendance depuis Césaire à « une écriture engagée, engagée dans le combat anticolonialiste, mais, en conséquence, engagée aussi hors de toute vérité intérieure, hors de la moindre esthétique littéraire ${ }^{10}$. L'heure n'est plus à l'unification d'un groupe conscient de lui-même contre l'Occident. D'une part, les auteurs usent d'un «nous » analytique : il ne s'agit plus d'unir les " nègres » autour d'une civilisation commune, mais de se proclamer créole en décomposant les différentes composantes - européennes, africaines, asiatiques ${ }^{11}$ - de cette identité. D'autre part, en mobilisant l'œuvre de Césaire, les auteurs soulignent un " héritage spécifique ${ }^{12}$ de la culture antillaise : à travers la figure du «père fondateur », un champ littéraire autonome transparait ; et les auteurs se positionnent par rapport à lui. Ils cherchent à se distinguer de leur prédécesseur. La situation culturelle s'est complexifiée: l'écrivain antillais n'est plus uniquement dans une posture d'opposition à 1'Occident; il se démarque aussi des héritages littéraires caribéens.

Edouard Glissant se situe à l'articulation de ces deux postures : la représentation anticoloniale, où l'écrivain se charge de prendre la parole au nom du peuple, et l'analyse

\footnotetext{
${ }^{9}$ L.C. Picanço, Vers un concept de littérature nationale martiniquaise, New York / Bern / Berlin, Peter Lang, 2000, p. 20.

${ }^{10}$ J. Barnabé / P. Chamoiseau / R. Confiant, Eloge de la créolité, Paris, Gallimard, 1993 (1989), pp. 20-21.

${ }^{11}$ Voir ibid., p. 13.

${ }^{12}$ V. Bonnet, « Les traces intertextuelles ou l'affirmation d'un champ littéraire franco-antillais », in R. Fonkoua / P. Halen /

K. Städtler (dir), Les Champs littéraires africains, Paris, Kathala, 2001, p. 137.
} 
postcoloniale, où l'écrivain prend position par rapport au peuple, mais aussi par rapport à des traditions culturelles mieux établies. Dans ce changement de posture, la place du sujet par rapport au collectif est redéfinie. En effet, lorsque les auteurs de l'Eloge de la créolité parlent de retrouver une « vérité intérieure ». Ils expriment ainsi l'exigence de parler depuis un point de vue antillais (et non plus nègre ou universel), comme le souhaite Edouard Glissant ; mais la formule renvoie aussi à une subjectivité individuelle assumée par l'écrivain.

Glissant cherche lui aussi à se distinguer de Césaire en construisant une œuvre originale. Dans l'Intention poétique, il décrit la poésie de Césaire comme une « co-naissance au monde et de soi-même ${ }^{13}$. Selon Glissant, le poète du Cahier d'un retour au pays natal rend possible la conscience de soi : le lyrisme lui permet de faire entendre sa voix. En même temps, il désigne ce qui empêchait cette voix, le racisme colonial. De ce fait, l'expression de soi coïncide avec l'expression collective et au-delà avec des valeurs universelles, humanistes qui fondent la commune humanité du poète, de son peuple et du reste du monde : «aussi le poète assume-t-il non seulement sa naissance, et celle de son peuple, mais la connaissance toute, le vœu actuel de l'humain $»^{14}$. Le subjectif et le collectif sont inséparables : ils apparaissent ensemble immédiatement.

En traitant de la poésie césairienne, l'auteur de L'Intention poétique adopte une toute autre posture. Le sujet glissantien est un sujet critique : l'écrivain se tient à distance de la figure de Césaire et de sa vision du monde. Glissant insiste en effet sur les ruptures. D'une part, il cherche ce qui fait la spécificité des êtres plutôt que des identités abstraites. D'autre part, s'il reconnait « une perfection dans les premiers ouvrages de Césaire ${ }^{15}$, il se différencie de cette œuvre exemplaire. La première page du chapitre de L'Intention poétique consacré à Césaire fait part des réticences de Glissant :

« on peut être moins ouvert à cette autre nécessité, d'une sorte de libération de l'être fondamental. On contestera la valeur de la replongée aux forces obscures, on niera qu'il faille ici une identification postulée avec la terre et l'arbre $»^{16}$.

L'auteur reproche ici à Césaire ce qu'il nommera dans le Discours antillais la «pratique du Détour $»^{17}$. Le poète cherche à revenir à une essence fondamentale, à la terre mère africaine. Mais ce retour, aux yeux de Glissant, est devenu impossible à cause de la coupure historique qu'a représentée la Traite. Césaire se trompe donc dans l'adéquation poétique qu'il postule

\footnotetext{
${ }^{13}$ E. Glissant, L'Intention poétique, Paris, Gallimard, 1997 (1969), p. 138.

${ }^{14}$ Ibid., p. 139.

${ }^{15}$ Ibid., p. 137.

${ }^{16}$ Idem.

${ }^{17}$ E. Glissant, Le Discours antillais, Paris, Seuil, 1981, pp. 34-36.
} 
entre le poète, son peuple et des communautés plus abstraites (la civilisation négro-africaine, l'universel).

L'auteur de L'Intention poétique reconnaît à Césaire sa place et son rôle, mais il l'inscrit aussi dans une histoire littéraire. En effet, selon lui, «ces œuvres de Césaire prendront leur plein sens quand elles seront intégrées dans une littérature du pays même $»^{18}$. Il prend donc ses distances avec une œuvre qui fut avant tout un $«$ moment $»{ }^{19}$.

Le sujet glissantien ne vient donc pas au monde en découvrant et en révélant un peuple et une humanité ; il prend position dans un champ littéraire, et plus largement dans une histoire et une culture. Il ne s'y confond pas, mais il y prend place sur un mode critique.

\section{De la représentation à la réflexion}

Le rapport de l'écrivain à la représentation change alors du tout au tout. Le poète césairien «se voit dans ses frères $»^{20}$ : il y a une relation de transparence absolue qui permet au sujet poétique et au représentant politique de se confondre. Aimé Césaire représente son peuple à l'Assemblée nationale et à la mairie de Fort-de-France parce qu'il n'y a aucun obstacle à sa compréhension du peuple.

Il en va différemment pour Edouard Glissant. L'essayiste glissantien ne renonce pas entièrement à la représentation, mais elle est l'objet d'une médiation. Il n'y a plus de transparence entre l'écrivain et le peuple. La compréhension n'est plus immédiate : elle nécessite une analyse. Aussi les Antillais n'apparaissent-ils pas directement à Edouard Glissant : il les appréhende à travers leur discours. C'est ainsi qu'on peut comprendre le titre de l'ouvrage qu'il publie en 1981 à partir de sa thèse d'Etat : Le Discours antillais. Dans ce livre, l'auteur multiplie les citations: les discours historiques y côtoient les énoncés prosaïques de la vie quotidienne ; les discours pédagogique, psychiatrique, historiographique, géographique, littéraire sont tour à tour essayés à propos des Antilles. Edouard Glissant montre un discours éclaté entre différents locuteurs, différentes situations sociales; tous ces énoncés constituent le «discours antillais». L'essai recueille des fragments de discours disparates qui forment la trame de la société antillaise. L'essayiste ne représente pas directement sa communauté ; il la reflète à travers ses mots, il la cite.

Edouard Glissant trouve dans cette approche médiate de la réalité antillaise une démarche qui s'accorde à sa vision du monde. En effet, il s'oppose à tout processus

\footnotetext{
${ }^{18}$ E. Glissant, L'Intention poétique, op. cit., p. 143. Sauf indication contraire, l'auteur souligne.

${ }^{19}$ Ibid., p. 138.

${ }^{20}$ Idem.
} 
d'unification au nom du Même pour préférer le Divers qui «signifie l'effort de l'esprit humain vers une relation transversale, sans transcendance universaliste $»^{21}$. La communauté antillaise, à ses yeux, met en cause un mode de pensée occidental. La société antillaise ne peut pas, en effet, se concevoir comme une société unifiée ; on ne peut la saisir qu'à travers une diversité de particularités. Les Antilles ne sont donc pas réductibles à l'Europe, à l'Afrique ou à une vision transcendante de l'humanité; elles ne se réduisent à aucune abstraction. Les Antillais sont d'abord pris dans la réalité d'une histoire et d'une géographie que l'auteur s'attache à faire affleurer en en saisissant les reflets dans le « discours antillais ». Cette réalité et, par conséquent, ce discours sont eux-mêmes divers et l'écrivain se doit de rendre compte de cette diversité.

Son rôle pourtant ne se réduit pas à celui de scribe consignant des paroles sans avoir prise sur elle. Dans les «Introductions » du livre, l'auteur présente son projet et rejette une forme de texte qui se fonde sur le Même : «l'édit», qui fixe des réalités dans un travail de synthèse subsumant la réalité des phénomènes derrière une définition ou une essence unique. Aussi l'essayiste cherche-t-il à «quitter l'édit, pour trouver [sa] parole $»^{22}$. Le «discours antillais » n'est pas uniquement un objet d'étude ; l'essayiste y participe, l'expression désigne aussi le livre lui-même. L'écrivain ne cherche pas seulement à consigner des paroles éparses ; il produit lui-même un discours fragmentaire qui s'accorde à la réalité qu'il observe et à laquelle il appartient. Il ne peut plus appréhender la communauté de manière immédiate, parce que la communauté se construit dans la médiation : il s'accorde donc à ce processus dans son écriture, y trouvant sa poétique et sa voix propres.

Edouard Glissant adopte une démarche similaire dans Poétique de la Relation. L'essai s'ouvre par un chapitre relatant «l'expérience du gouffre $»^{23}$ : celle de la traversée transatlantique de la Traite, où la cale du bateau négrier est à la fois un espace de mort et de rupture et le moment d'une naissance, tragique, à un nouveau monde qui sera celui de la culture antillaise. Cette expérience est celle d'un «non-monde où $t u$ cries $»^{24}$. L'essayiste fait porter son attention sur la voix des esclaves qu'il tente d'inscrire dans son texte. Cependant, l'emploi de la deuxième personne dans ce passage est alors ambigu. Glissant cherche-t-il à abolir la distance qui le sépare de l'esclave enfermé dans la cale, qui devient alors comme un double de l'essayiste ? Ou bien au contraire le « tu » instaure-t-il une distance ?

\footnotetext{
${ }^{21}$ E. Glissant, Le Discours antillais, op. cit., p. 190.

${ }^{22}$ Ibid., p. 14.

${ }^{23}$ E. Glissant, Poétique de la Relation, Paris, Gallimard, 1990, p. 19.

${ }^{24}$ Ibid., p. 18 (Nous soulignons).
} 
Selon Glissant, le cri doit donner naissance à la parole qui permet de lui conférer « une durée nouvelle, ancrée aux émergences des peuples $»^{25}$. Le cri est fragment, tout entier dans une souffrance et une douleur qu'il exprime; la parole permet d'articuler le cri à une histoire et à une géographie, à ouvrir l'imaginaire sur d'autres espaces et d'autres temps. La parole permet donc à la fois de se connaître, de prendre conscience du cri - elle a une dimension réflexive - et de créer des liens avec les autres. La parole ne se limite pas à l'expression d'une douleur ou d'un manque ; elle ouvre à la réflexion.

L'auteur de Poétique de la Relation ne fait pas que porter la voix des esclaves transbordés; il la réfléchit et l'articule à un ailleurs. L'écriture permet au « je », qui demeure discret dans le premier chapitre du livre, de mettre en relation le «tu» et le «nous». L'essayiste s'adresse à l'esclave : il ne représente pas son cri, mais il y trouve un reflet de luimême. L'esclave est une figure de la souffrance, mais le travail de réflexion en fait aussi le symbole de la mise en relation de plusieurs espaces - Afrique, Amérique, Europe. Le parcours de cet esclave est donc au croisement de plusieurs continents; il symbolise à la fois la douleur et la diversité qui donnent naissance à la société antillaise. En articulant le cri en une parole, Edouard Glissant passe du «tu» au «nous », se positionne au sein d'un espace social. En effet, la réflexion sur la figure et le corps meurtri de l'esclave débouche sur une conscience sociale et historique, sur une conception de soi à l'intérieur d'un espace antillais particulier et ouvert sur le monde :

« Nous nous connaissons en foule, dans l'inconnu qui ne terrifie pas. Nous crions le cri de poésie. Nous barques sont ouvertes, pour tous nous les naviguons. $»^{26}$

La démarche réflexive de l'essayiste permet au sujet de se situer dans une double perspective, historique et géographique. D'une part, il retrouve les traces de son passé et ouvre son existence présente à la mémoire collective de l'esclavage. D'autre part, il se situe dans un paysage, la Martinique, où il décèle des traces venues d'autres continents. Ainsi, l'essayiste se situe par rapport au temps et à l'espace, par rapport à la communauté, mais cette situation n'est pas une fixation. Au contraire, l'essayiste pratique une écriture critique et réflexive pour déjouer toute attitude essentialiste. Si l'écrivain se situe, il ne s'assigne pas à résidence.

\section{Le « voyage du dedans », l'errance au dehors}

\footnotetext{
${ }^{25}$ E. Glissant, Le Discours antillais, op. cit., p. 19.

${ }^{26}$ E. Glissant, Poétique de la Relation, op. cit., p. 21.
} 
Tout d'abord, la découverte de soi implique un «voyage du dedans » : l'écriture d'Edouard Glissant s'appuie, comme l'a montré Romuald Fonkoua, sur une forme de nomadisme ${ }^{27}$. L'essayiste découvre sa situation en arpentant son pays, comme par exemple dans ces chapitres symétriques de Poétique de la Relation où la réflexion de l'essayiste suit ses errances et ses promenades sur deux plages martiniquaises ${ }^{28}$.

Ensuite, l'ancrage dans un lieu n'empêche pas le voyage et la découverte de l'ailleurs. $\mathrm{Au}$ contraire il la rend possible. En effet, Glissant énonce ce principe : "Que l'étant est relation, et qui parcourt ${ }^{29}$. Selon l'auteur de Traité $d u$ Tout-Monde, il est impossible de remonter à une essence des choses et des êtres. Or l'existence est faite de mouvements : ces mouvements font que des êtres et des pays différents se rencontrent. Ces rencontres créent des « lieux communs », des espaces où il est possible de reconnaître à la fois l'étrangeté et la familiarité d'un lieu ou d'individu. Ainsi, l'auteur se reconnait parfaitement dans son lieu de naissance en Martinique, mais il lui est aussi possible de communiquer avec un autre lieu, tout différent, par un jeu de ressemblances. Par la magie de la comparaison et de la métaphore, sa mangrove natale peut rappeler la végétation des bords du Nil :

« Je suis ce pays de mangrove au Lamentin en Martinique où j'ai grandi et en même temps, par une infinie présence imperceptible, qui ne conquiert rien sur l'Autre, cette rive du Nil où les roseaux tournent à bagasse ainsi que des cannes à sucre. $»^{30}$

La Terre magnétique est emblématique de cette situation paradoxale d'Edouard Glissant. Le livre, qui porte sur l'île de Pâques, est publié dans une collection, dirigé par Glissant lui-même, qui vise à donner au public des ouvrages écrits par des écrivains partis sur un gréement, La Boudeuse, «à la rencontre de peuples accessibles par seule voie d'eau » ${ }^{31}$. La Terre magnétique semble donc s'apparenter au récit de voyage. Cependant, Glissant lui-même n'a pas pu faire le voyage sur l'île de Pâques, trop éprouvant pour sa santé ; l'ouvrage est donc écrit en collaboration avec Sylvie Séma, qui s'en rendue sur l'île et qui en a rapporté un matériel (vidéos, photographies, dessins) sur lequel Glissant s'appuie pour écrire. Ainsi le voyage est possible pour l'auteur resté chez lui. Il entre en relation avec un autre espace et une autre communauté, par le biais d'un tiers. Il rencontre Betty Rui, qui lui fait découvrir l'île, par le biais de vidéos ramenés par Sylvie Séma, sans jamais pouvoir dialoguer directement

\footnotetext{
${ }^{27}$ Voir R. Fonkoua, Essai sur une mesure du monde au XXème siècle, Edouard Glissant, Paris, H. Champion, 2002, pp. 9597.

${ }^{28}$ « La plage noire » et « La plage ardente », in E. Glissant, Poétique de la Relation, op. cit., pp. 135-142 et pp. 221-225.

${ }^{29}$ E. Glissant, Traité du Tout-Monde, Paris, Gallimard, 1997, p. 178.

${ }^{30}$ Idem.

${ }^{31}$ E. Glissant, La Terre magnétique, Paris, Seuil - Peuples de l'eau, 2007, Quatrième de couverture.
} 
avec elle. Il produit un texte en s'appuyant sur d'autres textes et sur des images. L'essai est donc le fruit d'une médiation complexe. L'essayiste voyage sans quitter son espace et sa situation; il se situe par rapport à un espace extérieur qu'il découvre en décrivant les différents médias qui rendent possible cette découverte. Le voyage n'est ni perte de soi, ni assimilation de l'autre : il est découverte de l'autre, de ses particularités, de ses dissemblances et de ses points communs avec le sujet.

La démarche d'Edouard Glissant entre alors en adéquation avec l'île de Pâques. Celleci apparaît comme un lieu à part, difficile d'accès. Mais son isolement est relatif puisqu'elle est l'objet de «toutes sortes d'exploitation radicales par toutes sortes de gens venus de l'extérieur, la déportation et l'esclavage et les massacres collectifs et les pandémies importées, et pour finir, [d']une lutte solitaire contre les formes infinies de dénaturation contemporaine $»^{32}$. L'histoire de l'île et les traces qu'elle laisse dans son paysage présentent donc des similitudes avec l'espace antillais, tel qu'il apparait dans Le Discours antillais ou Poétique de la Relation. L'île de Pâques est à la fois un espace isolé, à part, irréductible et solitaire et un lieu pris dans les dynamiques mondiales, connecté au reste du monde, similaire en cela à la terre natale de Glissant.

L'île est donc comme l'essayiste. C'est un espace qui ne bouge pas, couvert de «maisons fermées de partout, refuges barrés de murs de roches, où furent aussi confinés et préservés les poulets $»^{33}$, un espace dont la survie est lié à l'isolement, à la défense de ses particularités, de son paysage intérieur. Mais «l'île a la forme esquissée d'un bateau » : elle « bouge et navigue dans l'espace du Pacifique et sous la lune du grand triangle, en quête de la parole perdue $»^{34}$. Elle est animée d'un mouvement imperceptible qui la conduit peut-être à sa perte, du moins à sa transformation, mais qui la connecte dans le même temps au reste du monde.

L'écriture d'Edouard Glissant ne lui permet pas de faire corps avec son peuple, ni avec le monde : elle n'a pas la fulgurance d'une révélation qui ferait du poète le porte-parole d'un peuple, voire de l'humanité. Au contraire, il s'agit d'une écriture médiate: l'essayiste s'attache avant tout à se situer par rapport aux autres. Pour autant, il ne cherche pas à tout prix à s'en distinguer. Il analyse la part des autres - des autres individus, mais aussi des autres espaces et des autres époques - dans la construction de sa subjectivité. Et au cours de ce

\footnotetext{
${ }^{32}$ Ibid., p. 10.

${ }^{33}$ Ibid., p. 48.

${ }^{34}$ Ibid., pp. 48-49.
} 
processus, il retrouve la communauté : la sienne propre, celle de son espace natal, mais aussi d'autres sociétés avec lesquels cet espace est lié.

\section{Le « je » et le « tu » : subjectivité d'écrivain, subjectivité de lecteur}

\section{Dialogue subjectif : le rôle du lecteur dans l'écriture}

Le sujet ne prend pas soudainement conscience de son appartenance à une communauté ; il la découvre progressivement, par une analyse qui prend la forme d'un dialogue avec des figures issues de différentes époques et de différents espaces. La reconnaissance de la communauté est donc le fruit d'une démarche qui intègre l'altérité : le sujet découvre le « nous » en s'adressant à un « tu », qu'il s'agisse de l'esclave dans la cale du bateau négrier ou de l'habitante de l'île de Pâques. Mais plus profondément, le sujet se découvre et se construit lui-même en s'adressant ainsi à un interlocuteur. Le lecteur joue donc un rôle essentiel dans la construction du texte et de la subjectivité de l'essayiste.

Edouard Glissant s'adresse dans ses essais à des figures qu'il ne connait pas. Les esclaves du premier chapitre de Poétique de la Relation font l'expérience du gouffre, «trois fois noué à l'inconnu $»^{35}$. Il ne peut pas vraiment parler avec les habitants de l'île de Pâques, même si Betty Rui, qui le fait entrer dans l'univers de l'île, «voulut vraiment s'adresser à [lui], par le truchement de la minuscule caméra que Sylvie avait emportée $»^{36}$. La communication n'est jamais possible: le «gouffre», le temps ou la distance sépare l'essayiste de ses interlocuteurs. Ils sont donc liés à une histoire et à une expérience qui leur est propre et à laquelle l'écrivain ne peut avoir entièrement accès. Et à l'inverse, l'écrivain leur échappe - Betty Rui tente de l'imaginer de l'autre côté de la caméra, mais il lui demeure inconnu.

On se trouve donc face à une situation où le sujet découvre les autres en éprouvant leur opacité - et en lui opposant sa propre opacité. Pour le dire avec les mots de Judith Butler :

«L'autre m'expose son unicité, mais je lui expose également la mienne, et cela ne veut pas dire que nous sommes identiques, mais seulement que nous sommes liés l'un à l'autre par ce qui nous différencie, c'est-à-dire par notre singularité. ${ }^{37}$

Cependant, la philosophe met en perspective cet échange :

\footnotetext{
${ }^{35}$ E. Glissant, Poétique de la Relation, op. cit., p. 18.

${ }^{36}$ E. Glissant, La Terre magnétique, op. cit., p. 28.

${ }^{37}$ J. Butler, Le Récit de soi, Paris, PUF, 2007 (2005), p. 34 (Traduction : B. Ambroise / V. Aucouturier).
} 
" "cette" exposition singularisante [...] constitue une condition collective, nous caractérisant tous également, ne réinstallant pas seulement le "nous", mais établissant également une structure interchangeable au cœur de la singularité. ${ }^{38}$

Le dialogue avec un autre dont on respecte l'opacité permet de retrouver la communauté ; il est la démarche qui fonde la conscience collective. Plus encore, il permet de mettre l'accent sur le caractère fondamental de l'échange avec autrui pour la conscience de soi. En effet, je ne découvre mon unicité qu'en l'exposant à un autre qui m'expose la sienne : je ne me construits donc, en tant que sujet singulier, qu'à travers un échange et un dialogue avec un autre. Ma singularité et mon unicité de sujet ne préexistent pas à ma rencontre avec l'autre : elles se constituent au cours de cette rencontre.

Le rôle de ses interlocuteurs est donc primordial dans la construction de la subjectivité d'un individu, le rôle de ses lecteurs tout aussi important pour la construction de l'écrivain. Edouard Glissant met en scène ce processus à deux niveaux dans ses essais.

Il y a tout d'abord des situations de dialogues qui préexistent au texte et qui lui donne naissance : l'essayiste construit ses idées en discutant avec d'autres individus avant d'écrire son texte. Plutôt que de masquer ces échanges, l'essayiste va au contraire les exhiber, soulignant par là le rôle qu'ont joué les premiers lecteurs (ou les premiers auditeurs) : ils apparaissent comme des contributeurs à l'élaboration du texte. Ainsi certains fragments des essais de Glissant ont d'abord pris la forme de conférences données à des public divers. L'auteur, non seulement mentionne ce premier état du texte, mais il intègre aussi les séances de questions qui ont suivi les conférences. Ainsi, dans Le Discours antillais ou dans Introduction à une Poétique du Divers, la pensée de Glissant s'élabore par précisions et corrections successives, suite aux questions qui lui sont posé par ses interlocuteurs, nettement individués et nommés.

Dans Mémoires des esclavages, un dialogue avec une habitante de Bordeaux, suite à une conférence, suscite un développement portant sur l'effacement des traces de l'esclavage dans les grandes villes françaises de la côte atlantique. Ce dialogue n'est pas l'occasion pour le descendant d'esclaves de faire la leçon à la descendante d'esclavagistes. En effet, alors que la dame remercie l'écrivain des précisions qu'il lui a donné, l'essayiste remercie à son tour la dame pour sa question, qui lui a «enseigné sur la mémoire et sur ces exigences » ${ }^{39}$. Le dialogue a suscité l'écriture du texte ; il a aussi fait affleurer des souvenirs. La rencontre et

\footnotetext{
${ }^{38}$ Ibid., p. 35.

${ }^{39}$ E. Glissant, Mémoires des esclavages, Paris, La Documentation française / Gallimard, 2007, p. 77.
} 
l'échange avec un interlocuteur ont construit en partie le sujet : en indiquant à la Bordelaise ce qu'elle devait ne pas oublier, Glissant s'est lui-même trouvé des souvenirs et une place par rapport à Bordeaux et à ses habitants. Le dialogue lui a permis de découvrir un nouvel espace, un souvenir commun à lui et à son interlocutrice : «Vous m'avez indiqué qu'il est un lieu dans tant de chemins, une traverse, où se rencontrer $»^{40}$.

$\mathrm{Au}$ terme de cette logique du dialogue constructif, on trouverait les cas de collaborations littéraires qui ponctuent le parcours d'Edouard Glissant. L'essayiste ne se contente plus alors de discuter avec un autre en amont de son travail d'écriture; il s'associe à quelqu'un qui devient le co-auteur du texte. Le travail avec Sylvie Séma sur La Terre magnétique en témoigne. La co-écriture de deux opuscules avec Patrick Chamoiseau, Quand les murs tombent (2007) et L'Intraitable Beauté du monde (2009), en constitue la formule la plus radicale : les deux voix se mélangent sans qu'on puisse démêler ce qui vient de l'un et ce qui vient de l'autre ; les livres sont constitués de l'échange de deux subjectivités.

Le second de ses ouvrages permet de voir comment joue la mise en scène du rôle du lecteur à un deuxième niveau, à l'intérieur même du texte. Il ne s'agit plus de mettre en scène un premier lecteur, qui a transformé le texte et qui y apparait donc ; cette fois, le lecteur est une figure du livre qui y joue un rôle rhétorique et conceptuel.

L'Intraitable Beauté du monde est une «adresse à Barack Obama »: le lecteur est désigné dans le sous-titre. Les deux auteurs choisissent donc pour interlocuteur le premier président «noir » des Etats-Unis. Ils s'adressent à un individu, porteur d'un projet politique précis, mais ils voient surtout en lui la reconnaissance du phénomène de "créolisation des sociétés modernes, qui s'oppose aux traditionnelles poussées de l'exclusive ethnique, raciale, religieuse et étatique des communautés actuellement connues dans le monde $»^{41}$. Ce n'est donc pas tant le programme politique du président américain qui intéresse les deux essayistes. Ils s'adressent à Barack Obama parce que son élection change, à leurs yeux, la façon de concevoir la représentation politique.

Barack Obama n'est pas le représentant politique d'un groupe fondé sur une identité fixe. Edouard Glissant et Patrick Chamoiseau commencent en effet par rappeler le caractère fragmenté, divers et « créole » de la société américaine. En effet, les Noirs américains

\footnotetext{
${ }^{40}$ Idem.

${ }^{41}$ P. Chamoiseau / E. Glissant, L'Intraitable Beauté du monde - Adresse à Barack Obama, Paris, Galaade, 2009, p. 4.
} 
« sont entrés dans la puissance étatsunienne, comme un de ses fondements, mais aussi comme un de ses manques. Comme une puissance et comme un manque et comme la plus précieuse des fragilités. Ils sont en nous. Ils sont en vous, monsieur. $»^{42}$

Barack Obama se construit dans son rapport à la diversité de la société américaine. Il ne représente pas un peuple défini de manière positive; au contraire, il construit sa stature politique sur une altérité constitutive des Etats-Unis: il prend en charge l'histoire contradictoire et complexe de la société, il doit articuler la puissance actuelle du pays avec les souffrances qui sont à son origine.

L'interlocuteur devient une sorte de personnage du texte, qui permet aux essayistes de se construire : ils s'adressent à lui parce qu'ils se situent eux aussi dans cette histoire de souffrances et de manque. Barack Obama n'est pas leur représentant - ni Patrick Chamoiseau ni Edouard Glissant ne sont citoyens américains - mais un interlocuteur. Il représente moins un peuple que la possibilité d'un dialogue, la capacité pour un dirigeant politique à entendre «le cri $d u$ monde, la voix des peuples et le chant joyeux ou meurtri des pays ${ }^{43}$. Il leur permet de se constituer à la fois comme manque, sujets minorisés issus d'une histoire douloureuse, et comme puissance, partie prenante d'une société et d'un monde qu'ils contribuent à édifier et où une place peut leur être reconnue.

Cependant, Barack Obama n'est pas à proprement parler le lecteur du livre. Très prosaïquement, L'Intraitable Beauté du monde est écrit en français et publié dans une maison d'édition parisienne : le président des Etats-Unis n'est donc pas, de toute évidence, son lecteur-cible. Par ailleurs, le livre est construit sur une alternance entre des passages en italiques où les essayistes s'adressent directement, à la deuxième personne, à Barack Obama, et des passages en caractères latins où il devient l'objet du texte. Edouard Glissant et Patrick Chamoiseau parlent alors de lui à la troisième personne, pour analyser la signification de son parcours et de son accession à la présidence américaine. Le lecteur change selon les passages du livre. Et si Barack Obama, interlocuteur et lecteur fictif du texte, les construit en sujets d'une écriture politique qui ne soit plus fondée sur la représentation, il est aussi l'objet d'analyses et d'interprétations qui expliquent ce rôle joué par Obama pour les auteurs. Le lecteur construit donc le sujet de l'écriture ; mais l'essayiste construit aussi son lecteur. Ou, plus exactement, l'essayiste s'adresse successivement à plusieurs lecteurs (tantôt Barack

\footnotetext{
${ }^{42}$ Ibid., pp. 1-2.

${ }^{43}$ Ibid., p. 4.
} 
Obama, tantôt le lecteur français): le sujet de l'énonciation se construit à l'interstice de plusieurs dialogues.

\section{Lignes de fuite}

L'essai glissantien s'inscrit dans une tradition montaignienne en ce qui concerne le rapport au lecteur. En effet, l'adresse au lecteur qui ouvre les Essais de Montaigne est ambivalente. D'une part, l'auteur déclare que son livre est destiné à des «parens et amis $»^{44}$. Pourtant, en s'adressant à un « lecteur » indéfini, l'auteur semble viser un public plus large que celui de ses proches : les Essais relèvent à la fois de la conversation familière et de l'œuvre d'art visant à la postérité.

Dans les essais d'Edouard Glissant, on retrouve cette ambivalence : le lecteur y est une figure changeante; l'essayiste s'adresse à plusieurs lecteurs en même temps. Les dialogues avec des interlocuteurs divers donnent aux livres par endroits le ton de la conversation. En d'autres endroits, les textes tendent à la communication scientifique. Par exemple, les analyses sur l'évolution d'une expression créole dans Le Discours antillais ${ }^{45}$ semblent s'adresser d'abord à des sociolinguistes; dans Introduction à une Poétique du Divers ${ }^{46}$, les commentaires sur les concepts développés par Gilles Deleuze et Félix Guattari paraissent concerner au premier chef les philosophes. En d'autres passages, Glissant propose des réflexions à portée très générale, comme pour un lecteur abstrait. Les premières pages de Philosophie de la Relation proposent ainsi des réflexions qui vaudraient pour «toutes les littératures du monde $»^{47}$.

En s'adressant tour à tour à des lecteurs variés, l'essayiste refuse de s'assigner un rôle nettement défini. Il apparait à la fois comme le protagoniste de conversations familières, qui réagit aux propos d'un autre, comme un auteur scientifique, suivant un protocole d'observation et s'appuyant sur une bibliothèque donnée, comme un penseur qui propose des vues générales et originales sur le monde et la littérature.

\section{L'écrivain-lecteur}

En transformant sans cesse la figure du lecteur, l'essayiste ne se montre pas moins redevable à lui : il lui est nécessaire de prévenir ses attentes et d'y répondre. En même temps,

\footnotetext{
${ }^{44}$ Montaigne, Essais - Livre I, Paris, PUF - Quadrige, 1992 (1965 - 1580-1588), p. 3 (Edition de P. Villey).

${ }^{45}$ E. Glissant, Le Discours antillais, op. cit., pp. 349-351.

${ }^{46}$ E. Glissant, Introduction à une Poétique du Divers, Paris, Gallimard, 1996, pp. 59 et sq.. On retrouve en de nombreux autres passages des essais de Glissant des commentaires de la philosophie de G. Deleuze et F. Guattari.

${ }^{47}$ E. Glissant, Philosophie de la Relation, Paris, Gallimard, 2009, p. 11.
} 
il se met à chaque fois lui-même en position de lecteur : l'essayiste est semblable à celui à qui il s'adresse, il est lui aussi lecteur.

Georg Lukacs, dans la lettre-préface à L'Ame et les formes, insiste sur une spécificité de l'essai : «l'essai parle toujours de quelque chose qui possède déjà une forme $»^{48}$. L'essayiste se distingue en ceci du poète : il ne crée pas une forme nouvelle, il reconfigure un objet, un ouvrage ou une œuvre d'art qui a déjà été créé. « Il ne fait que leur conférer une organisation nouvelle et ne forme rien de nouveau à partir d'une absence de forme $»^{49}$.

Et Glissant en effet apparait toujours peu ou prou dans ses essais comme un lecteur, qui reconfigure l'objet de son étude. C'est le cas dans Le Discours antillais. L'auteur s'y fait le lecteur d'un discours social qu'il reconfigure dans son livre. Il en préserve la diversité en adoptant une forme éclaté et fragmentaire. Mais il oriente la lecture de cette diversité : elle n'est plus contingente, mais la forme de l'essai permet de l'éclairer et de l'expliquer. En effet, le discours antillais tel qu'il est lu dans l'essai de Glissant est fragmenté pour deux raisons. D'une part, les lacunes de la conscience historique antillaise conduisent à « une sorte de nonsavoir à travers quoi est tenté l'effort de nier l'avoir totalisateur et corrodant de l'Autre $»^{50}$. En oubliant une partie de leur histoire, les Antillais n'ont pas conscience de ce qui les sépare et les singularise de la métropole. De ce fait, le discours social antillais ne peut pas se construire et il est voué à n'exister que par bribes. D'autre part, les Antilles sont un espace de diversité : la culture antillaise est constituée de différents apports que le discours n'amène jamais à la synthèse ; ils apparaissent dans leur diversité.

Par ailleurs, dans L'Intention poétique, Glissant lit les œuvres de grands poètes. Il reconfigure cependant tous ces textes puisque sa lecture est guidée par l'ambition de «dépasser l'ambition extatique de 1 'Un » ${ }^{51}$. Il cherche à travers différentes lectures à faire progressivement affleurer l'image du Divers ; il approche les œuvres en insistant sur les traces « du disparate et de l'opposé $»^{52}$ qu'elles portent en elles. L'essayiste dialogue avec des œuvres déjà en forme. Elles sont pour lui des interlocuteurs nécessaires pour construire sa démarche et pour se construire en tant qu'écrivain. Plus encore, dans son travail de reconfiguration de ces œuvres, il fait apparaître qu'elles sont elles-mêmes le fruit de rencontres et d'échanges.

\footnotetext{
${ }^{48}$ G. Lukacs, « Nature et forme de l'essai », in F. Dumont (ed.), Approches de l'essai, op. cit., p. 33. Traduction de D. Bohler / F. Hartweg.

${ }^{49}$ Idem.

${ }^{50}$ E. Glissant, Le Discours antillais, op. cit., p. 276.

${ }^{51}$ E. Glissant, L'Intention poétique, op. cit., p. 13.

${ }^{52}$ Ibid., p. 14.
} 
Glissant semble donc actualiser dans ses essais le genre du «texte-lecture », proposé par Roland Barthes ${ }^{53}$ et dont le modèle serait son $S / Z$. Ce genre n'est pas réductible à la critique universitaire ou scientifique ; il met en avant « une logique du symbole » :

« Cette logique-là n'est pas déductive, mais associative : elle associe au texte matériel (à chacune des phrases) d'autres idées, d'autres images, d'autres significations. " ${ }^{54}$

Glissant lit les œuvres en y faisant jouer des associations d'idées et de significations qui ouvrent les textes et dévoilent leur caractère divers et disparate. Dans ses essais, il lit un corpus en le faisant entrer en relation avec des considérations historiques, avec des paysages, avec d'autres textes. Il ne s'attache pas uniquement à décrire et interpréter les textes; il cherche à les éclairer à travers des éléments qui leur sont extérieurs.

Deux éléments cependant distinguent la pratique d'Edouard Glissant de celle de Roland Barthes. Tout d'abord, Barthes, à l'instar de Lukacs, maintient la séparation entre la lecture et la composition littéraire. Selon lui les règles de la composition sont rhétoriques et rationnelles et s'opposent à la logique d'association de l'écriture-lecture. Glissant a une autre conception de l'écriture. Dans Traité du Tout-Monde, il insiste en effet sur le caractère baroque de la création artistique dans les Amériques, qui se caractérise alors à ses yeux «par l'étendue, l'accumulation, la prolifération, la répétition ${ }^{55}$. L'écriture de Glissant passe donc moins par un travail de composition rhétorique - au sens classique où l'entend Barthes - que par un travail d'association et d'amplification, caractéristique d'une écriture libre et ouverte, celle de l'écriture-lecture.

La frontière entre littérature et critique littéraire, entre écriture et lecture s'estompe donc tout à fait chez Glissant. A tel point que, dans Philosophie de la Relation, l'avant-dernier chapitre consacré de Mahmoud Darwich prend la forme d'un poème. Glissant approche l'œuvre poétique du poète palestinien en s'intéressant au travail de l'écriture : le poème prend la forme d'une réflexion sur le travail langagier sur le «Nom» dans la poésie de Darwich ${ }^{56}$. Mais Glissant ne choisit pas la forme d'une prose développant un aspect de cette œuvre littéraire; il livre sa réflexion en vers, répondant aux vers arabes de Darwich. Ce passage est à la fois la lecture d'un poète et l'écriture d'un poème.

\footnotetext{
${ }^{53}$ R. Barthes, Le Bruissement de la langue, Paris, Seuil - Tel Quel, 1984, pp. 34 et sq.

${ }^{54}$ Ibid., pp. 34-35.

${ }^{55}$ E. Glissant, Traité du Tout-Monde, op. cit., p. 116.

${ }^{56}$ E. Glissant, Philosophie de la Relation, op. cit., p. 153.
} 
Mais c'est surtout sur la question de la subjectivité que l'opposition entre Glissant et Barthes apparait le plus nettement. En effet, pour Barthes, le texte-lecture n'est pas affaire de subjectivité. Pour lui, «toute lecture dérive de formes transindividuelles $»^{57}$. La lecture est guidée d'abord par des règles linguistiques et un imaginaire qui dépasse l'individu; le textelecture joue de ces règles et il est tout entier fait de ce jeu. Ce qui importe, ce n'est ni la communauté à travers laquelle les règles sont fixées, ni l'individu qui les subit; la lecture fait intervenir le plaisir et le corps, en deçà de l'individu et du sujet. Chez Barthes, il y a trois instances : l'individu sujet, qui est un leurre, est entièrement dominé et formé par les règles fixées par la communauté, la deuxième instance; en-deçà, il y a le corps, où une véritable singularité peut se loger et trouver son expression dans le plaisir. Le corps ne constitue pas une subjectivité puisqu'il est tout entier composé de sensations, il précède toute conscience de soi.

Chez Glissant, cette triade est reconfigurée :

«Trois fois l'œuvre concerne. En ce qu'elle est pulsion d'un groupe d'hommes: communauté ; en ce qu'elle se noue au vœu d'un homme : intention; en ce qu'elle est ouvrage et drame d'humanité qui continue ici : relation. $»^{58}$

Entre le pôle collectif et le pôle individuel, Glissant situe la relation. Cette notion renvoie au caractère disparate et divers, multiple de toute communauté et de tout individu. Barthes préférait dissoudre le sujet pour loger la singularité ailleurs, dans le plaisir du corps. Glissant, lui, réaffirme la singularité de l'individu; il la met en relation avec une communauté. Cependant, la subjectivité de l'individu n'est pas purement et simplement le produit de règles édictées par la communauté. La communauté en effet est diverse et en relation avec différents lieux du monde; la subjectivité individuelle se construit en dialoguant avec ces différents aspects. Et la littérature - lecture ou composition - permet de révéler la mise en relation d'un sujet avec la communauté et le monde qui l'entoure. Il lui est offert de reconfigurer le donné qui lui est imposé, de se construire en tant que sujet, en se situant par rapport aux autres, au groupe, au monde.

\section{Le « je » : un nouveau régime d'auctorialité}

\section{L'adéquation textelobjet}

\footnotetext{
${ }^{57}$ R. Barthes, Le Bruissement de la langue, op. cit., p. 35.

${ }^{58}$ E. Glissant, L'Intention poétique, op. cit., p. 25.
} 
Le rôle dévolu à l'auteur est donc entièrement repensé dans les essais d'Edouard Glissant. L'essayiste déjoue tous les protocoles d'écriture attendus. Dans L'Intention poétique, il propose une critique littéraire à contre-courant: contre la sacralisation de l'écrivain et aussi contre la sacralisation du texte, il propose de «nou[er] à rebours le lien entre le monde et [l'œuvre] $»^{59}$, de réinscrire l'œuvre dans la relation que l'écrivain entretient avec le monde. Dans Le Discours antillais, il propose de repenser la périodisation historique en insistant sur la nécessité de prendre en compte le « discontinu réel » d'une histoire heurtée sous le « continu apparent» suscité par la tradition historique ${ }^{60}$. Dans La Terre magnétique, il perturbe la tradition de l'enquête anthropologique: il n'a aucun contact direct avec son « terrain », le dialogue qu'il entreprend avec ses « informateurs » est ouvertement médiat et opaque.

Glissant malmène donc différentes traditions de l'écriture du savoir. Il en remet en cause les règles pour proposer une écriture qui se fonde sur une adéquation à son objet d'une part, et sur le libre-arbitre du sujet d'autre part. En effet, Glissant perturbe le jeu de la critique littéraire dans L'Intention poétique pour échapper à des lectures qui, en se focalisant sur «l'CEuvre », restent prisonnière de l'imaginaire de «l'Un » ${ }^{61}$, qui n'arrivent pas à prendre en compte le Divers. Or sa situation d'écrivain antillais rend absolument nécessaire cette attention au Divers: la poétique de l'Un s'est construite dans la culture occidentale et l'écrivain martiniquais, " étranger dans [son] étrangeté » ${ }^{62}$ est sommé de proposer autre chose, de prendre en compte l'altérité qui constitue sa littérature. Dans Le Discours antillais, Glissant conteste la périodisation traditionnelle parce qu'elle conduit à « découper l'histoire de la Martinique sur le modèle de 1'histoire de France $»^{63}$. Il lui incombe donc de trouver un autre modèle qui permette de prendre en compte les différentes composantes historiques de la société martiniquaise.

\section{Le libre-arbitre de l'auteur}

Cependant, l'écriture n'est pas simplement soumise à son objet : le sujet intervient lui aussi pour justifier la conduite de son écriture. Ecrire une critique littéraire ou une histoire qui serait proprement antillaise risquerait de privilégier le pôle collectif en en faisant un absolu. L'individu risquerait de ne plus y trouver sa place. Mais, en insistant sur l'imperfection des

\footnotetext{
${ }^{59}$ Ibid., p. 19.

${ }^{60}$ E. Glissant, Le Discours antillais, p. 157.

${ }^{61}$ E. Glissant, L'Intention poétique, op. cit., p. 15.

${ }^{62}$ Ibid., p. 26.

${ }^{63}$ E. Glissant, Le Discours antillais, op. cit., p. 155.
} 
œuvres humaines dans L'Intention poétique ${ }^{64}$ et sur le discontinu de l'histoire dans Le Discours antillais ${ }^{65}$, Edouard Glissant propose une écriture du fragment. Il procède alors par association et par développement : ce n'est pas l'objet de l'essai qui dicte la méthode à l'essayiste, il la lui évoque.

Dans L'Intention poétique, l'essayiste cherche une méthode de lecture qui lui permette de rendre compte de l'altérité et de la diversité qui anime toute littérature. Il fait alors se succéder les lectures de différents textes, tirés de la littérature française et mondiale. L'essai est animé par le vœu d'une nouvelle manière de lire. Mais il n'est pas tout entier guidé par l'idée de fournir une lecture antillaise ou américaine de la littérature mondiale. En passant d'un auteur à l'autre, Glissant suit son humeur et ses envies : il répond à une « obligation simple : d'ouvrir et de ravir le corps des connaissances. De choisir ou d'élire parmi le proféré. De nommer ceux qu'il aime ou qu'il a fréquentés ${ }^{66}$. L'écriture s'adapte à l'objet de l'essai, mais l'essayiste adapte aussi son objet à son écriture, à ses goûts et à ses affects.

Ce faisant, il préserve la relation : non pas uniquement le pôle collectif ou uniquement le pôle individuel, mais une manière pour l'individu de comprendre le monde qui l'entoure et qui le construit en s'adaptant à lui et en l'adaptant à lui.

« Chacun a ses raisons d'aller à cette écoute et ces manière différentes servent à changer ce bruit du monde que tous en même temps nous entendons ici-là. $\rangle^{67}$

Le sujet prête attention à un objet qu'il choisit de traiter; il adapte alors son écriture à cet objet, tel qu'il le conçoit et tel qu'il le vit. Par l'écriture s'établit ainsi une relation entre le monde et le sujet, elle donne naissance à «des lieux communs que nous apprenons à partager $»^{68}$. L'écriture de Glissant permet de rendre lisible et visible le processus de la relation qui s'offre ainsi à un tiers, le lecteur. L'écriture est à la fois la relation d'un sujet avec le monde et d'un sujet avec un lecteur : elle crée ainsi une médiation entre le lecteur et le monde, saisi dans l'originalité d'une vision et d'une pensée.

\section{Un sujet sensible}

L'écriture de l'essai passe donc par une dimension affective : elle s'articule à la sensibilité de l'écrivain. Ainsi, Une nouvelle région du monde s'ouvre sur la description d'un

\footnotetext{
${ }^{64}$ E. Glissant, L'Intention poétique, op. cit., pp. 18-19.

${ }^{65}$ E. Glissant, Le Discours antillais, op. cit., p. 157.

${ }^{66}$ E. Glissant, L'Intention poétique, op. cit., p. 51.

${ }^{67}$ E. Glissant, Traité du Tout-Monde, op. cit., p. 17.

${ }^{68}$ Idem.
} 
paysage marin. Le sujet est situé dans ce paysage de tempête. Il est constitué des sensations qu'il éprouve face à ce paysage. Après avoir décrit la «vive alliance de la lumière et de l'obscurité » qui caractérise le littoral où il se trouve, le sujet se définit en des termes qui reprennent ses deux caractéristiques visuelles et contrastées du paysage : «ils m'ont alloué l'éclat, et j’habitais l'obscur ${ }^{69}$. Le paysage est relayé dans un collectif (un «ils») qui assigne une place au sujet - où il n'entre pas entièrement. Mais le sujet est aussi celui qui construit le paysage : tout un réseau de comparaisons et de métaphores dans ce passage présente le paysage «comme [...] la trame d'un texte $»^{70}$, celui que l'essayiste est en train d'écrire.

Cette interaction du sujet et du monde, de l'essayiste antillais et de son univers devient un lieu commun pour lui et le lecteur à travers la dimension sensible du texte. La description rend l'espace visible pour le lecteur : il peut s'y référer à partir de sa propre expérience corporelle des couleurs et des formes que décrit Glissant. Dans le même temps, la description se dénonce comme artefact par le jeu de métaphorisation : le paysage est d'abord un texte. In fine, le texte est le lieu commun par le biais duquel le sujet de l'écriture fait le lien entre lui, le monde et son lecteur.

\section{Conclusion}

Edouard Glissant se méfie dans ses essais de la représentation : les textes n'offrent aucune synthèse qui permettrait au sujet de se montrer garant d'un peuple ou d'une identité, face à un lecteur qui serait entièrement extérieur. Au contraire, Glissant privilégie l'analyse et la médiation. Ces deux stratégies d'écriture coïncident avec une vision du monde. Plutôt que de définir des identités, Glissant cherche en effet à dévoiler des processus.

Tout apparait alors en interaction. Le sujet du texte se constitue dans ses relations avec sa communauté ; mais la communauté elle-même s'élabore dans des interactions dynamiques avec le reste du monde. L'essayiste se construit aussi dans sa relation avec son lecteur : le « je » apparaît alors comme la mise en relation d'un «tu », d'un " nous » et du reste du monde. Glissant déjoue donc les logiques d'opposition pour proposer une écriture où le sujet de l'écriture, plutôt que représentant, se fait passeur ou médiateur, entre les individus et les communautés, entre le lecteur et le monde.

\footnotetext{
${ }^{69}$ E. Glissant, Une nouvelle région du monde, Paris, Gallimard, 2006, p. 11.

${ }^{70}$ Idem.
} 
Un nouvel imaginaire politique correspond donc à cette écriture. Il a quelque chose d'utopique puisque, en refusant la représentation pour la médiation, l'essayiste se refuse à exercer toute forme de pouvoir. Il se construit en sujet politique d'un monde idéalement parfaitement égalitaire. Ce monde ne correspond certes pas à la réalité des rapports de force existant, que Glissant décrit et dénonce dans ses essais. Mais l'œuvre littéraire, en proposant une autre façon de concevoir les relations interindividuelles et internationales, appelle à une autre façon de penser le monde et la politique. Il reste sans doute à inventer les moyens de donner à cette vision une efficacité politique.

Florian ALIX 